\title{
Bone-specific circulating miRNA profile changes over an 8-week repeated sprint training protocol
}

Giovanni Lombardi ${ }^{1}$, PhD; Veronica Sansoni ${ }^{1}, \mathrm{PhD}$; Silvia Perego ${ }^{1}$, PhD; Gianluca Vernillo ${ }^{2,3}, \mathrm{PhD}$; Mattia Bonzanni ${ }^{4}$, MSc; Giampiero Merati ${ }^{2,5}$, MD; Andrea Barbuti ${ }^{4,6}, \mathrm{PhD}$; Antonio La Torre ${ }^{2}$, MSc; Giuseppe Banfi ${ }^{1,7}, \mathrm{MD}$

${ }^{1}$ Laboratory of Experimental Biochemistry \& Molecular Biology, I.R.C.C.S. Istituto Ortopedico Galeazzi, Milano, Italia ${ }^{2}$ Dept. of Biomedical Sciences for Health, Università degli Studi di Milano, Milano, Italia ${ }^{3}$ CeRiSM, Research Center for Sport, Mountain and Health, Università degli Studi di Verona, Verona, Italia ${ }^{4}$ Dept. of Biosciences, Università degli Studi di Milano, Milano, Italia ${ }^{5}$ IRCCS Fondazione Don Carlo Gnocchi, Milano, Italia UniSR ${ }^{6}$ Centro Interuniversitario di Medicina Molecolare e Biofisica Applicata (CIMMBA), Università degli Studi di Milano, Milano, Italia ${ }^{7}$ Università Vita-Salute San Raffaele, Milano, Italia

\section{INTRODUCTION}

BACKGROUND: MicroRNAs (miRNAs) are single-stranded 18-22 nucleotides-long non-coding RNAs solving a key role in the finetuning of gene expression. They binds the mRNA 3'-UTR sequences and inhibit translation by inducing the enzymatic degradation of the target mRNA. In the last few years, the identification of circulating miRNAs, as surrogate markers of tissue miRNA activities, has grown so as to speculate their use as disease markers [1]. Bone metabolism is regulated by specific microRNAs and changes in their expression are associated with bone metabolic disorders and fracture risk [2].

Exercise is a main determinant of bone metabolism [3]; however, different training protocols differently affect bone [4]. Up to now, however, little is known about the effects of exercise on bonespecific fracture-risk associated with circulating miRNA levels. AIM: We studied the effect of a 8-week high-intensity training (HIT) protocol on circulating levels of selected miRNA associated with fracture-risk in elderly [5].

\section{METHODS}

SUBJECTS: 9 males (age 24.3 $\pm 3.7 \mathrm{y}$; BMI $22.1 \pm 1.7 \mathrm{~kg} / \mathrm{m}^{2}$ ) performed repeated sprint (RS) training 3 times/week (18x15m sprints with $17 \mathrm{~s}$ of passive recovery) for 8 weeks.

miRNA ANALYSES: Blood was taken at start (T0) and after 4 (T1) and 8 weeks (T2). miRNA-enriched total RNA was extracted from plasma with miRCURY ${ }^{\mathrm{TM}}$ RNA Isolation Kit (Exiqon). Extraction efficiency was checked through 3 synthetic oligos (spike-ins: Sp2, Sp4, Sp5) added at recommended concentrations. RT and Real-Time PCR were performed with miRCURY LNA ${ }^{\text {TM }}$ Universal RT microRNA PCR and Universal cDNA synthesis kit II (Exiqon), using Sp6 and Cel39 as internal controls. Real-time PCR was performed by using specific miRCURY LNA ${ }^{\text {TM }}$ microRNA PCR primers (Exiqon). miRNAs tested: miR-21-5p, miR-23a-3p, miR-24-3p, miR93-5p, miR-100-5p, miR122-5p, miR-124-3p, miR-125b-5p, miR-148a-3p, miR-637. Relative expressions were calculated by the $2^{-\Delta \Delta C T}$ method. Among the suggested reference miRNAs, miR425-5p was chosen as housekeeping since more stable in this setting. Hemolysis was checked by the miR-23a-to-miR-451 $\Delta$ CT ratio (positive if $>7$ ).

STATISTICS: Changes over time where tested by Friedman's test. Significance level was set at $\mathrm{p}<0.05$ (GraphPad Prism v6.01, GraphPad SW Inc.).

\section{RESULTS}

In our cohort of young adult males, miR-637 and miR-124-3p were undetectable in plasma throughout the observation. miR-21-5p, miR-93-5p, and miR-125b-5p were not modified by the intervention. miR-23a-3p, miR24-3p, miR-100-5p showed a net decrease between T0 and T2. miR-122-5p and miR148a-3p significantly decreased from T0 to T1 and then increased to the baseline levels at T2. Interestingly, miR-100-5p increased at T1 and then, after 8 weeks, it decreased below the T0 levels. This was accompained by a very evident widening of the distribution of values.

\section{.} hsa-miR-21-5p

$$
\text { hsa-miR-23a-3p }
$$
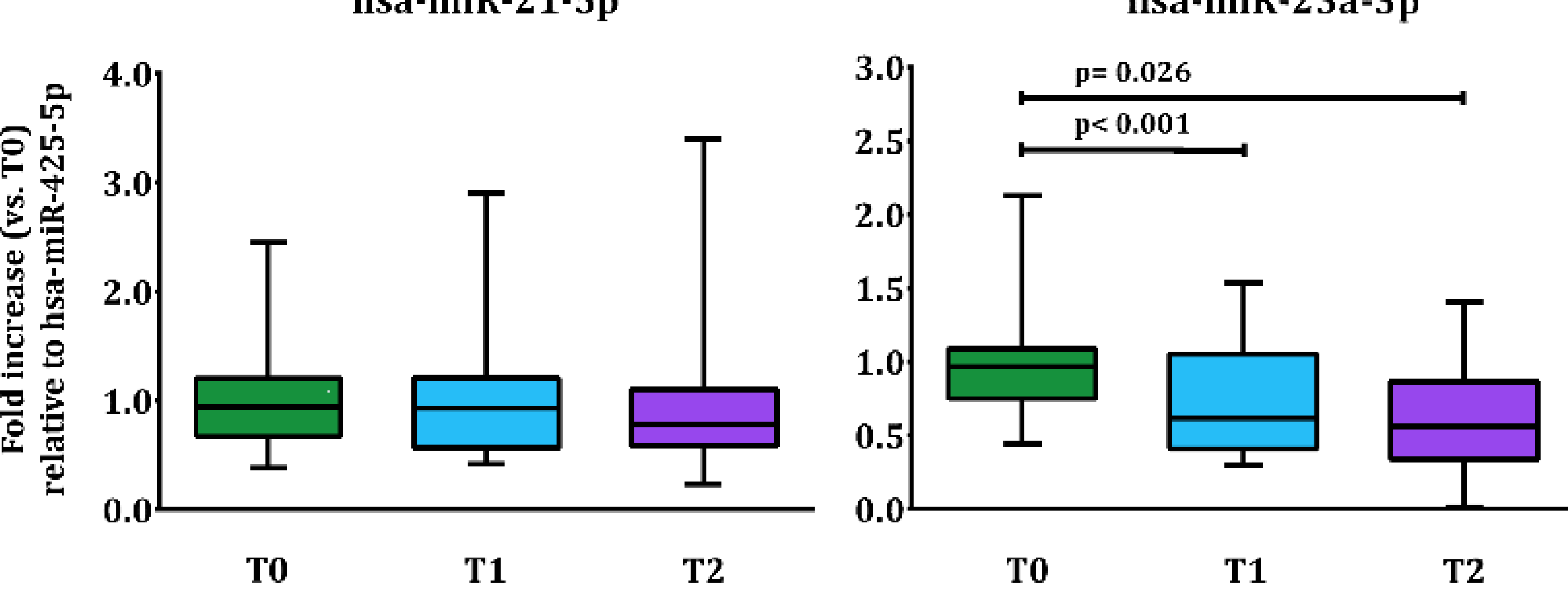
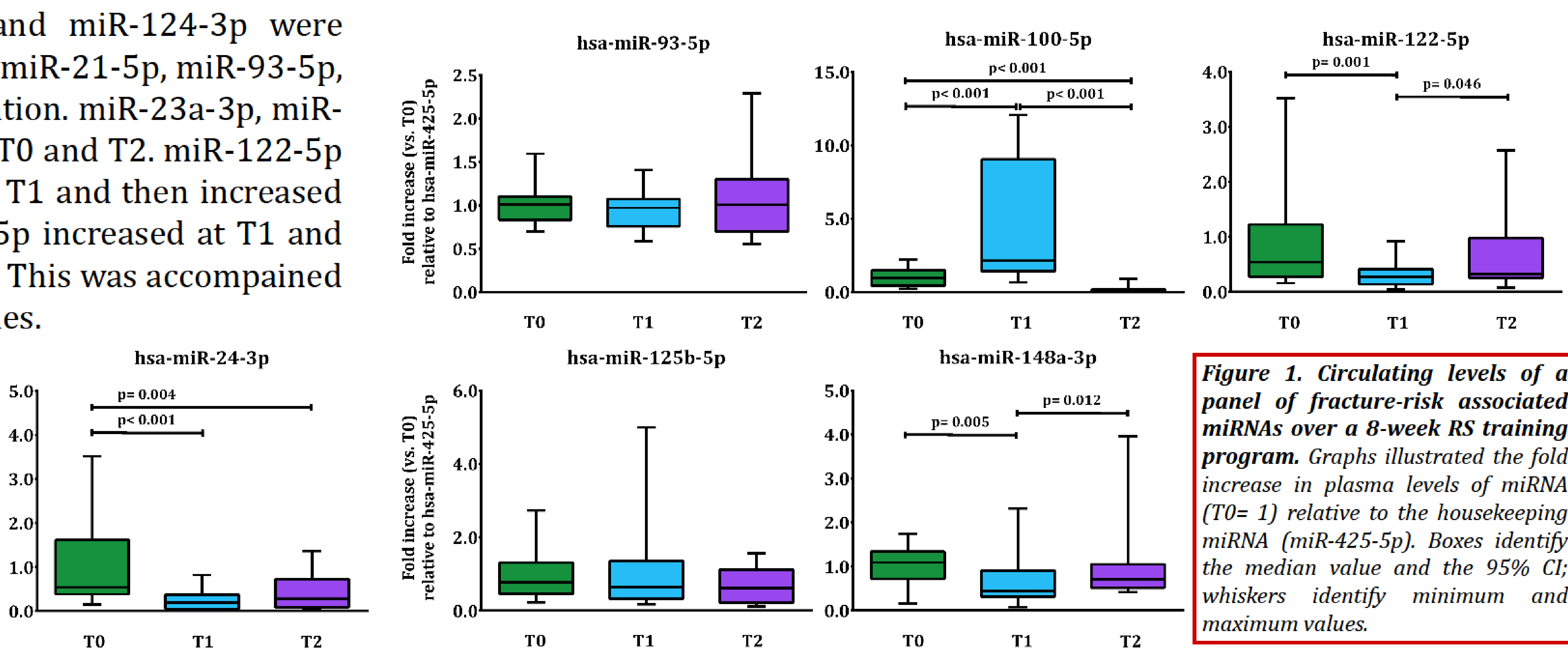

Figure 1. Circulating levels of panel of fracture-risk associated miRNAs over a 8-week RS training program. Graphs illustrated the fold increase in plasma levels of miRNA $(T 0=1)$ relative to the housekeeping miRNA (miR-425-5p). Boxes identify the median value and the $95 \%$ CI whiskers identify maximum values.

\section{CONCLUSIONS}

Altered expression of bone-specific miRNAs can lead to osteoporosis and fragility fractures. Increased circulating levels of miR-21-5p, miR-23a-3p, miR-24-3p, miR-93-5p, miR-100-5p, miR-122-5p, miR-124$3 p$, miR-125b-5p, miR-148a-3p were found in patients with osteoprotic fractures. miR-21-5p, miR-23a$3 p$, miR-24-3p, miR-100-5p, and miR-125b-5p expression was higher in osteoporotic bone tissues [5]. miR-637 affects the pre-osteoblast-to-osteoblast differentiation by inhibiting Osterix expression [2] High-impact exercises are effective in improving/maintaining bone mass, at least in women, from childhood to old age; on the contrary, the effect of HIT protocols on bone health are still debated [4].

The main findings of this study are:

- miR-124-3p and miR-637, normally detectable in plasma of elderly, were undetectable in our cohort of young adult males: this fracture-risk associated miRNAs could be also associated with aging.

- 8-week RS training protocol is sufficient to reduce the circulating levels of some fracture-risk associated miRNAs. miR-23a-3p, miR-24-3p, and miR-100-5p decreasing response lasted the whole observation, while miR-122-5p and miR-148a-3p acutely decreased in the first half and then recovered up to baseline.

A short-term HIT protocol effectively improves the circulating profile of fracture risk-associated miRNA. Hence, continuative RS training in youth could be an effective strategy to prevent fractures in older ages.

\section{REFERENCES}

1. Li Z, Rana TM. Nature Rev Drug Discov 2014; $13: 622-38$

2. Gámez B, Rodriguez-Carballo E, Ventura F. $\boldsymbol{J}$ Mol Endocrinol 2014;52:R179-97

3. Lombardi G, Sanchis-Gomar F, Perego S, Sansoni V, Banfi G. Endocrine Epub

4. Xu J, Lombardi G, Jiao W, Banfi G. Sports Med. Epub.

5. Chen J, Qiu M, Dou C, Cao Z, Dong S. Drug Development Res 2015;76:235-45

\section{Contact information:}

Giovanni Lombardi, Ph.D.

Laboratory of Experimental Biochemistry \& Molecular Biology I.R.C.C.S. Istituto Ortopedico Galeazzi

Via Riccardo Galeazzi, 4 -20161, Milano, Italia

giovanni.lombardi@grupposandonato.it

\section{ACKNOWLEDGMENTS}

Study supported by the Italian Ministry of Health and the Italian Ministry of University, Education \& Research THE AUTHORS HAVE NO COMPETING INTERESTS 